\title{
Erratum to: A new F-18 labeled PET tracer for fatty acid imaging
}

\author{
Fabian Demeure, MD, ${ }^{\mathrm{a}, \mathrm{b}}$ Manuel D. Cerqueira, $M D,{ }^{\mathrm{c}}$ Michel Hesse, $\mathrm{PhD},{ }^{\mathrm{d}}$ \\ David Vancraeynest, $M D, P h D,{ }^{\mathrm{a}, \mathrm{b}}$ and Véronique Roelants, $\mathrm{MD}, \mathrm{PhD}^{\mathrm{d}, \mathrm{e}}$ \\ a Pôle de Recherche Cardiovasculaire, Institut de Recherche Expérimentale et Clinique, Université \\ catholique de Louvain, Brussels, Belgium \\ ${ }^{b}$ Division of Cardiology, Cliniques Universitaires Saint-Luc, Brussels, Belgium \\ c Department of Nuclear Medicine, Imaging Institute, Cleveland Clinic, Cleveland Clinic Lerner \\ College of Medicine of Case Western Reserve University, Cleveland, $\mathrm{OH}$ \\ ${ }^{d}$ Nuclear Medicine Department, Cliniques Universitaires Saint-Luc, Brussels, Belgium \\ e Pôle d'Imagerie moléculaire, Radiothérapie et Oncologie, Institut de Recherche Expérimentale \\ et Clinique, Université catholique de Louvain, Brussels, Belgium
}

doi: $10.1007 / \mathrm{s} 12350-014-0046-7$

\section{ERRATUM TO: J NUCL CARDIOL DOI 10.1007/S1 2350-014-0012-4}

The following acknowledgement was omitted from the published article:

Acknowledgments Patient images were obtained under informed consent from a research study sponsored by FluoroPharma Medical. The study's principal investigator is Olivier Gheysens MD PhD, Department of Nuclear Medicine, UZ Leuven, Belgium.

The online version of the original article can be found under doi: 10.1007/s12350-014-0012-4.

Reprint requests: Véronique Roelants, $\mathrm{MD}, \mathrm{PhD}$, Nuclear Medicine Department, Cliniques Universitaires Saint-Luc, Avenue Hippocrate, 10, 1200 Brussels, Belgium; veronique.roelants@ uclouvain.be

J Nucl Cardiol 2015;22:398.

$1071-3581 / \$ 34.00$

Copyright (c) 2014 American Society of Nuclear Cardiology. 